\title{
Altered squat movement pattern in patients with chronic low back pain
}

\author{
Magdalena Zawadka',A-D ${ }^{1,}$, Jakub Smołka ${ }^{2, B-D \oplus}$, Maria Skublewska-Paszkowska ${ }^{2, C-D \oplus}$, \\ Edyta Łukasik ${ }^{2, C-D \oplus}$, Grzegorz Zieliński ${ }^{1, D \oplus}$, Aleksandra Byś ${ }^{1, D} \oplus$, Piotr Gawda ${ }^{1, A, E-F} \oplus$ \\ ${ }^{1}$ Department of Sports Medicine, Faculty of Health Sciences, Medical University, Lublin, Poland \\ 2 Department of Computer Science, Faculty of Electrical Engineering and Computer Science, University of Technology, \\ Lublin, Poland \\ A - Research concept and design, B - Collection and/or assembly of data, C - Data analysis and interpretation, \\ $D$ - Writing the article, $E$ - Critical revision of the article, $F$ - Final approval of article
}

Zawadka M, Smołka J, Skublewska-Paszkowska M, Łukasik E, Zieliński G, Byś A, Gawda P. Altered squat movement pattern in patients with chronic low back pain. Ann Agric Environ Med. 2021; 28(1): 158-162. doi: 10.26444/aaem/117708

\begin{abstract}
Introduction and objective. Low back pain (LBP) is a problem which causes human suffering and entails costs for treatment. Chronic LBP is considered a civilization diseases in developed countries and one of the major public health problem worldwide. Squatting is a part of such daily activities as sitting down, standing up or lifting objects. In this study, hip, knee, and ankle joint motions during squatting are analyzed in the sagittal plane. The aim of the study was to find a relationship between the function of the lower limb during squatting and LBP incidence.

Materials and method. Eleven healthy subjects and eight subjects suffering from low back pain were enrolled in tes study. Participants performed squat while maintaining heel contact with the ground throughout the movement. The participants attempted to make a squat as deep as possible. Kinematic data were captured using a Vicon optical motion capture system. Results. The low back pain group had a greater range of motion of the hip and knee with reference to the ankle joint motion, compared to the control group. There was no statistically significant difference in ankle dorsiflexion between groups. Control group reached maximal ankle dorsiflexion at higher point of descent than LBP group.

Conclusion. The findings support the hypothesis that the strategy of movement is different in subjects with and without back pain. They may suggest that subjects with low back pain in maximal squat use the hip joint and knee joint to a greater extent than subjects without back pain, with comparable ankle dorsiflexion. Clinicians should take into consideration the influence of ankle motion and timing on exercise biomechanics in persons suffering from low back pain.
\end{abstract}

Key words

Low back pain, ankle joint, knee joint, hip joint, public health

\section{INTRODUCTION}

Low back pain (LBP) is a problem which causes human suffering and entails costs for treatment. About $80 \%$ of adults have experienced LBP at least once in their lifetimes [1]. Thus, chronic LBP is considered as a one of the civilization diseases in developed countries. Clinicians and researchers seek to determine the sources and causes of LBP based on posture and movement tests, and to provide guidelines for the management of LBP [2]. In LBP, a limited range of hip motion can lead to compensation in the lumbopelvic area during lower limb movement [3]. It was also observed that different movement patterns occurred during forward bending in subjects with and without $\operatorname{LBP}[4,5]$. This confirms a biomechanical connection between low back disorders and the function of other joints during dynamic tasks. Manual material handling and lifting activities have been associated with LBP [6]. One of the techniques involved is lifting with flexed knees (squat technique) [7]. Squatting is also part of such daily activities as sitting down and standing up.

Squatting exercises are an important component of programmes in physical therapy and in sports training [8]. Weight-bearing exercises are more functional than open

Address for correspondence: Magdalena Zawadka, Department of Sports Medicine, Faculty of Health Sciences, Medical University, Lublin, Poland

E-mail: magdalenazawadka91@gmail.com

Received: 13.12.2019; accepted: 30.01.2020; first published: 27.02.2020 chain exercises without weights. They have been included in several protocols to treat musculoskeletal disorders, such as patellofemoral pain syndrome, iliotibial band syndrome, and other dysfunctions [9-12]. Squatting is also used for examination of muscular activation and motor control [13, 14] because it is a complicated activity generating hip flexion, knee flexion, and ankle dorsiflexion. There is connection between low back pain and the lower limbs muscle activity [15]. Many papers have investigated the squatting technique and its parameters and provide a comparison of kinematic data during the squatting exercise in various groups: male and female [16] and healthy older adults [17]. The effectiveness of motion during squatting was analyzed on the basis of the healthy group and of patients with various diseases [18-21].

The foot and ankle joints are the most distal elements of the closed kinetic chain during functional activities. The trunk position and activity of the muscles are linked with the lower extremity function in the kinematics closed chain movement, such as lifting and squatting, when a foot is stabilized on the ground $[22,23]$. Previous studies suggest that restrictions in the available ankle range of motion (ROM) may cause changes in sagittal and frontal plane kinematics at the knee joint [1, 21]. The foot and ankle position may be also considered as a contributing factor for changes of pelvic tilt [24]. The foot and ankle may influence the position of the upper segments of the lower limb and the trunk [23]. Moreover, previous studies have documented that the descent phase of a squat is 
initiated with an activity of the tibialis anterior [11]. However, the aforementioned papers lack computed correlation of the joint angles in the lower limbs during the full squat. The authors of the presented study hypothesize that the ankle is the element which determines the strategy of the squatting movement. The current study presents results indicating correlation between the clinical symptoms (in this case LPB) and the movement parameters of the lower limbs.

\section{OBJECTIVE}

The aim of the study was to analyze hip knee and ankle joint motions during squat in the sagittal plane, and to find a relationship between lower limbs joints ROM, squat depth and LBP incidence. It was hypothesized that the range of motion in lower limbs joints are different in subjects with and without LBP. Therefore, different movement patterns were observed.

\section{MATERIALS AND METHODS}

Subjects. Nineteen subjects: 11 healthy ( 3 female and 8 male) and 8 with LBP (4 female and 4 male) participated in the study (Tab. 1). The LBP group had physical examinations before the investigation and consisted of young subjects (students recruited from a local university) with pain persisting for longer than 6 weeks. In order to assess the pain, the Numerical Rating Scale (NRS) containing 11 degrees of pain intensity was used. The scale was from 0 - 10, 0 indicating absence of pain and 10 for maximum pain. The characteristics were elaborated on the basis of the answers given by the patients during examination. Subjects who reported the onset of back pain secondary to trauma were excluded. Also excluded were the subjects who had undergone spinal surgery, radicular symptoms, or specific posture training as part of treatment or any other methods of low back pain physical therapy. The third exclusion factor included subjects with pain greater than $5 / 10$ on the NRS scale in order to limit the influence of avoiding pain on the movement performance. The study included only participants who had experienced low back pain for longer than 6 weeks to eliminate subjects with acute LBP. Volunteer students took part in the study as a asymptomatic control group.

Table 1. Group characteristics

\begin{tabular}{lcc}
\hline Variables & $\begin{array}{c}\text { LBP }(\mathrm{n}=8) \\
\text { mean (DS) }\end{array}$ & $\begin{array}{c}\text { Controls }(\mathrm{n}=11) \\
\text { mean }(\mathrm{SD})\end{array}$ \\
\hline Age (years) & $22.43(1.51)$ & $23.00(2.24)$ \\
\hline Body lenghth $(\mathrm{cm})$ & $171.86(9.14)$ & $174.43(11.12)$ \\
\hline Body weight $(\mathrm{kg})$ & $61.00(11.60)$ & $65.88(8.07)$ \\
\hline BMl $\left(\mathrm{kg} / \mathrm{m}^{2}\right)$ & $20.47(1.83)$ & $21.77(1.99)$ \\
\hline
\end{tabular}

Approval for the research was obtained from the Medical University of Lublin and Lublin University of Technology Ethical Committees, Nos. 6/2015 and KE-0254/331/2015, respectively. All participants were informed about the study procedures in detail and signed informed consent prior to the measurements.
Instrumentation. 3D kinematic data were captured using a Vicon optical motion capture system equipped with 8 NIR T40S cameras (2 cameras mounted on each wall of the motion recording room), and 2 reference Bonita cameras on tripods. Vicon Nexus 2.0 software was used for data collection and post-processing. Data were sampled at $100 \mathrm{~Hz}$. Each patient had 39 markers placed on his/her body, as specified in the Plug-in Gait Model documentation. The markers were fixed to the skin using Vicon double-sided tape. Each patient was measured in compliance with the Plugin Gait Model specification (leg length, knee width, ankle width, shoulder offset, elbow width, wrist width, hand width, weight and height), so that the subjects created in the Vicon Nexus software could be scaled and calibrated. All motion recordings were made in a room without daylight access.

The Plug-in Gait Model computes the subject kinematics and kinetics data. The model is divided into 4 rigid body segments: kinematic lower body, kinematic upper body, kinetic lower body and kinetic upper body. The kinematic models define the rigid body segments and allow the calculation of joint angles between segments. The kinetic models are responsible for computing moments of inertia of the segments which are used in turn for computing segment reactions. For the purpose of this study, 3 computed angles (hip, knee and ankle) were used to evaluate the patient's mobility (Fig. 1).

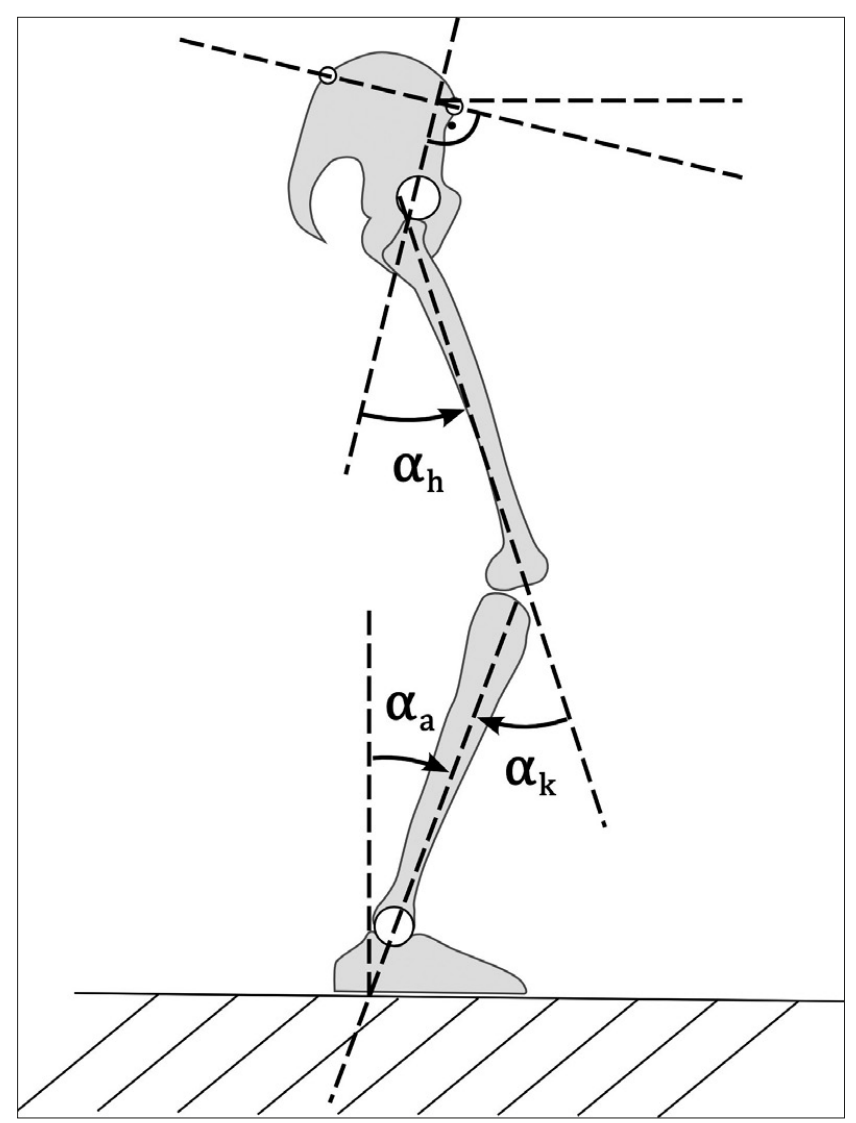

Figure 1. The angles under consideration

Procedure and data capture. Each participant's initial position was standing, with hands along the trunk, feet at shoulder width apar, and toes at a $15-20$ degree angle. The patient was then instructed to extend both arms anteriorly, perform a full squat, and return to the initial standing 
position. During the squat, the participants were barefoot and maintained heel contact with the ground throughout the movement. The participants tried to perform as deep a squat as possible.

The squat was considered to have been performed correctly if there was no loss of balance and the motion was fluent. Each participant was allowed to start the exercise whenever he/she felt comfortable. Participants had 3 practice trials before the recording was made. No time restriction was set.

Data analysis. Recorded 3D data were post-processed and each marker properly labelled. Gaps in marker trajectories were filled-in. The Plug-in Gait dynamic model was executed. The recorded and generated data were then exported to a C3D file that contained markers' positions and model output for each frame of the recording. Since this study examined relations between different joint angles, a custom piece of software was created to compute a set of selected joint angles for a given set of 'control' joint angles. The software was written in $\mathrm{C}++$ using B-TK (Biomechanical Toolkit) and Eigen libraries.

Only the results of the descent phase of the squat were analyzed in this study. The angles under consideration are depicted in Figure 1, where: $\alpha_{\mathrm{a}}$ - ankle angle, $\alpha_{k}-$ knee angle, $a_{h}$ - hip angle. Because controls at the beginning of the movement (at the initial position) had more than 5 degrees of ankle dorsiflexion, knee and hip angles were analyzed only when the range of ankle dorsiflexion was from 10-30 degrees. The ankle was selected as the referent joint for the movement of other joints because foot-ankle complex is the last, fixed segment in the closed kinematic chain during squatting. The retro-reflective marker S2 (second sacral spinous process) height was used to measure the depth of the squat. The maximum value of the ankle joint angle was determined for each patient $\left(\max _{\alpha a}\right)$. The difference $\Delta \mathrm{h}$ was calculated as shown in equation 1 , where parameters $h_{\min }$ and $h_{\max }$ respectively stand for the minimal and maximal height of S2 reached during the squat. The custom software computed $\Delta \mathrm{h}$ for all moments of time when the vertical $(\mathrm{Z})$ coordinate of S2 expressed in centimetres had an integer value.

$$
\Delta h=h_{\max }-h_{\min }
$$

This parameter was used to define a new indicator $(F)$ for each prticipant. Its definition is presented in equation 2 . This is the quotient of the squat depth and the maximal ankle angle that the patient reached.

$$
F=\frac{\Delta h}{\max _{\alpha_{\mathrm{a}}}}
$$

Data analysis was conducted using Statistica software (ver. 13.1) and significance level was set at $p=0.05$. The ShapiroWilk test was used to evaluate the normal distribution for each investigated group. Due to lack of normal distribution, a U-Mann-Whitney non-parametric test for 2 independent samples was used.

\section{RESULTS}

The LBP group had a significantly greater angle of knee flexion during maximal squat for 10,15, 20 degrees of ankle dorsiflexion ( $\mathrm{p}<0.01 ; \mathrm{p}<0.05$ respectively). Hip angle was also significantly greater in LBP group than in controls in 20 and 25 degrees of ankle dorsiflexion ( $\mathrm{p}<0.05 ; \mathrm{p}<0.01$, respectively). In the same ankle dorsiflexion, hip and knee joints angles were generally greater in LBP participants, compared to controls (Fig. 2).

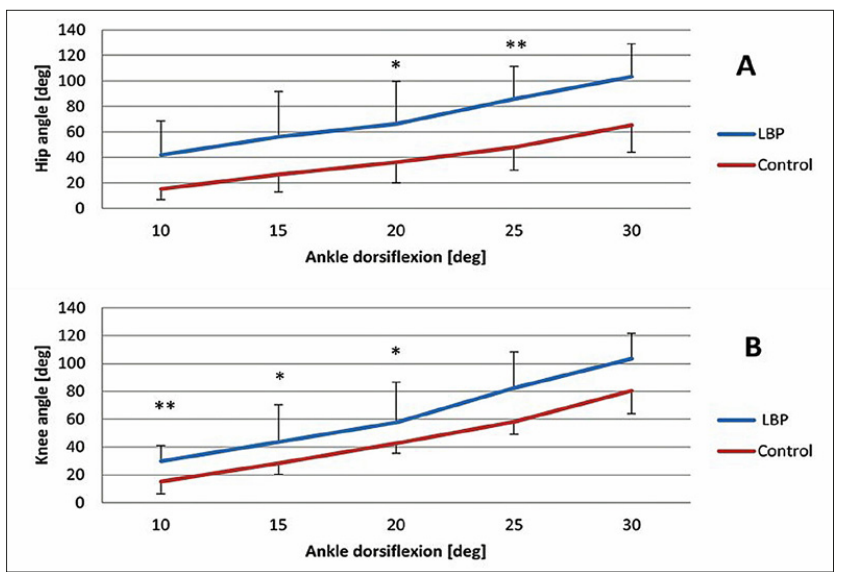

Figure 2. Hip flexion $(A)$ and knee flexion $(B)$ during maximal squat with reference to ankle joint dorsiflexion. ${ }^{*}-\mathrm{p}<0.05 ;{ }^{*}{ }^{*}-0<0.01$.

The control group demonstrate greater maximal range of ankle dorsiflexion of about 4 degrees, compared with LBP group. However, this data was not statistically significant $(\mathrm{p}=0.36)$ (Tab. 2). Difference in maximal squat depth, defined as $\operatorname{Max} \Delta h$, was not significant between groups. There were statistically significant differences in the depth of squat when the ankle reached maximal angle of dorsiflexion $(p=0.03$ in left ankle and $p=0.02$ in both ankles) (Tab. 2). Control group reached maximal ankle dorsiflexion at a higher point of descent $(83.31 \%$ of max delta $\mathrm{h}$ ) than the LBP group ( $93.84 \%$ of max delta h). Indicator F was higher for the LBP group in all cases (left, right and both) $(\mathrm{p}<0.05)$.

Table 2. Maximal ankle joint dorsiflexion, squat depth and indicator $F_{a v}$

\begin{tabular}{lccc}
\hline & $\begin{array}{c}\text { Control Group } \\
\mathrm{N}=11 \\
\text { Mean }(S D)\end{array}$ & $\begin{array}{c}\text { LBP Group } \\
N=8 \\
\text { Mean }(S D)\end{array}$ & $\begin{array}{c}\mathrm{P} \\
\text { value }\end{array}$ \\
\hline $\begin{array}{l}\text { Left ankle } \\
\left.\text { max } \mathrm{a}_{\mathrm{a}}{ }^{\circ}\right]\end{array}$ & $41.44(10.91)$ & $36.34(6.94)$ & 0.48 \\
\hline $\begin{array}{l}\text { Right ankle } \\
\left.\text { max } \mathrm{a}_{\mathrm{a}}{ }^{\circ}\right]\end{array}$ & $39.06(8.56)$ & $35.53(5.81)$ & 0.46 \\
\hline $\begin{array}{l}\text { Both ankles (average) } \\
\left.\text { max } \mathrm{a}_{\mathrm{a}}{ }^{\circ}\right]\end{array}$ & $40.25(9.39)$ & $35.93(5.66)$ & 0.36 \\
\hline Max $\Delta \mathrm{h}[\mathrm{mm}]$ & $580.00(110.72)$ & $618.75(87.25)$ & 0.43 \\
\hline Left $\Delta \mathrm{h}[\mathrm{mm}]$ & $490.91(60.57)$ & $581.25(90.94)$ & $\mathbf{0 . 0 3}$ \\
\hline Right $\Delta \mathrm{h}[\mathrm{mm}]$ & $475.45(96.58)$ & $580.00(114.14)$ & 0.05 \\
\hline Left and right (average) $\Delta \mathrm{h}[\mathrm{mm}]$ & $483.18(63.89)$ & $580.63(97.78)$ & $\mathbf{0 . 0 2}$ \\
\hline$F_{h}$ left side & $12.43(2.84)$ & $16.15(2.12)$ & $\mathbf{0 . 0 1}$ \\
\hline$F_{h}$ right side & $12.39(2.49)$ & $16.40(2.52)$ & $\mathbf{0 . 0 0 6}$ \\
\hline Left and right (average) $F_{h}$ & $12.41(2.27)$ & $16.28(1.91)$ & $\mathbf{0 . 0 0 6}$
\end{tabular}

Bold values indicate statistical significance $(p<0.05)$

max $a_{a}\left[{ }^{\circ}\right]$ - maximal ankle dorsiflexion angle

Max $\Delta h$ - maximal squat depth

Left $\Delta \mathrm{h}$ - squat depth when left ankle reached maximal dorsiflexion

Right $\Delta \mathrm{h}$ - squat depth when right ankle reached maximal dorsiflexion

$\Delta \mathrm{h}$ left and right - average squat depth for both ankles when they reached maximal dorsiflexion $F_{h}$ left side, $F_{h}$ right side, $F_{h}$ right and left indicator $F$ - squat depth when ankle reached maximal dorsiflexion divided by range of ankle dorsiflexion - for left ankle, right ankle and both of them, respectively. 


\section{DISCUSSION}

The current study shows that LBP and function of the lower limb during squatting can be related. These findings support the hypothesis that the movement pattern is different in subjects with and without LBP.

Previous studies report that limited ankle-dorsiflexion ROM during the squat resulted in the inability to achieve full knee flexion [21]. The presented study shows that the LBP group had a greater range of motion of the hip and knee with reference to the same ankle joint dorsiflexion, compared to control group. These findings may suggest that subjects with LBP in maximal squat use the hip and knee joint to a greater extent than subjects without LBP (hip dominant squat). Therefoer, they show different movement patterns during squatting than non-LBP subjects with the same angle in the ankle joint during motion. Mechano-receptors of ligaments of the ankle are one of the most important factors when the upper segments of the body are moved with the foot fixed on ground. Ankle proprioception is the regulatory mechanism for stabilization of the body $[25,26]$; therefore, deficits in the ankle complex proprioception in LBP can affect movement strategy during a dynamic task for keeping balance. How ankle proprioception is related to LBP occurrence can be a challenge for future research.

In this study there were no significant differences in the range of ankle dorsiflexion during squatting, when comparing the participants suffering from low back pain and those who were healthy. The study supports previous results which reported that the average ankle dorsiflexion required during a squat was $38.5 \pm 5.9^{\circ}[10,27]$.

To achieve larger angles in the hip and knee joints, backward displacement of the center of pressure (COP) has to occur. In this position, the trunk tilts forward to keep COP within the base of support. That position causes greater activity of the gluteus maximus as the strongest extensor of the hip $[28,29]$. Dorsiflexion of ankle and knee flexion displaces the thigh and shank anteriorly, favouring displacement of the COP towards the direction of the toe tip. This is a more balanced position than the hip-dominant squat observed in the LBP group. Kasuyama et al. reported that ankle dorsiflexion flexibility is strongly associated with the ability to assume the deep squat posture. When limitation in dorsiflexion is observed, it is necessary to move the centre of gravity forward by compensating with trunk and hip flexion [30]. Patients suffering from back pain demonstrated a compensation strategy to increase hip flexion with a stiffened lumbar spine in the sagittal plane during squatting [31]. Therefore, it is believed that the increased hip motion observed in LBP subjects is a compensation related to lumbopelvic dysfunction. How ankle joint motion during dynamic activities affects the motion of lower limb and pelvic joints needs to be investigated further.

Maximal squat depth proved to be comparable in both groups. However, the depth was different among the groups when descent to the point where ankle joint dorsiflexion was maximal was taken into account. This means that healthy controls reached a maximal range of ankle dorsiflexion in higher position during squat than LBPs. Participants suffering from back pain reached a maximal range almost at the deepest point of the descent. In the current study, the descent in millimetres by one degree of dorsiflexion in subjects with LBP was significantly greater compared to the control group. Differences observed in depth when maximal ankle dorsiflexion was reached may support previously reported schematic movement patterns in LBP patients [23]. A possible explanation of the presented findings can be more synchronous motion of the joints of the lower limbs in LBP patient during squatting.

The study has some limitations. First, the study was performed on a relatively small number of patients; therefore, the results must be interpreted with caution. Second, passive ankle ROM was not measured because the aim of the study was to examine the range of motion in a closed cinematic chain. As previously reported, the range of motion depends on the position [32], and it is different in weight-bearing and non-weight-bearing tasks [33]. Thus, the active and passive ranges of motion were not compared. Moreover, other authors consider that the squat can be used to examine the ankle dorsiflexion ROM [30]. More research on a larger sample size may explain the obtained results and confirm the authors' suppositions.

\section{CONCLUSIONS}

The obtained findings may suggest that subjects with low back pain in maximal squat use the hip and knee joints to a greater extent than subjects without back pain. This information may have implications for the proper executing of exercises during the rehabilitation of patients suffering from LBP. Patients with low back pain reached maximal ankle dorsiflexion in the lower position of squat than the controls. Clinicians should take into consideration the influence of ankle motion on exercise biomechanics in persons suffering from low back pain.

\section{Acknowledgments}

The study was not financed by any specific grant from funding agencies in the public, commercial, or not-for-profit sectors.

\section{REFERENCES}

1. Larivière C, Gagnon D, Loisel P. A biomechanical comparison of lifting techniques between subjects with and without chronic low back pain during freestyle lifting and lowering tasks. Clin Biomech Bristol Avon. 2002; 17(2): 89-98.

2. O'Sullivan P. Diagnosis and classification of chronic low back pain disorders: maladaptive movement and motor control impairments as underlying mechanism. Man Ther. 2005; 10(4): 242-55. https://doi. org/10.1016/j.math.2005.07.001

3. Kim S, Kwon O, Yi C, Cynn H, Ha S, Park K. Lumbopelvic motion during seated hip flexion in subjects with low-back pain accompanying limited hip flexion. Eur Spine J. 2014; 23(1): 142-8. https://doi.org/10.1007/ s00586-013-2973-4

4. Kim M, Yi C, Kwon O, Cho S, Cynn H, Kim Y, et al. Comparison of lumbopelvic rhythm and flexion-relaxation response between 2 different low back pain subtypes. Spine. 2013; 38(15): 1260-7. https:// doi.org/10.1097/BRS.0b013e318291b502

5. van Wingerden J-P, Vleeming A, Ronchetti I. Differences in standing and forward bending in women with chronic low back or pelvic girdle pain: indications for physical compensation strategies. Spine. 2008; 33(11): 334-341. https://doi.org/10.1097/BRS.0b013e318170fcf6

6. Marras WS, Lavender SA, Leurgans SE, Rajulu SL, Allread WG, Fathallah FA, et al. The role of dynamic three-dimensional trunk motion in occupationally-related low back disorders. The effects of workplace factors, trunk position, and trunk motion characteristics on risk of injury. Spine. 1993; 18(5): 617-28.

7. Mirakhorlo M, Azghani MR. Similarity of different lifting techniques in trunk muscular synergies. Acta Bioeng Biomech Wroc Univ Technol. 2015; 17(4): 21-9. 
8. Czaprowski D, Biernat R, Kędra A. Squat - Rules of Performing and Most Common Mistakes. Pol J Sport Tour. 2012; 19(1): 3-7. https://doi. org/10.2478/v10197-012-0001-6

9. Escamilla RF. Knee biomechanics of the dynamic squat exercise. Med Sci Sports Exerc. 2001; 33(1): 127-41.

10. Schoenfeld BJ. Squatting kinematics and kinetics and their application to exercise performance. J Strength Cond Res Natl Strength Cond Assoc. 2010; 24(12): 3497-506. https://doi.org/10.1519/JSC.0b013e3181bac2d7

11. Dionisio VC, Almeida GL, Duarte M, Hirata RP. Kinematic, kinetic and EMG patterns during downward squatting. J Electromyogr Kinesiol Off J Int Soc Electrophysiol Kinesiol. 2008; 18(1): 134-43. https://doi org/10.1016/j.jelekin.2006.07.010

12. Zwerver J, Bredeweg SW, Hof AL. Biomechanical analysis of the singleleg decline squat. Br J Sports Med. 2007; 41(4): 264-8; discussion 268. https://doi.org/10.1136/bjsm.2006.032482

13. Ageberg E, Bennell KL, Hunt MA, Simic M, Roos EM, Creaby MW Validity and inter-rater reliability of medio-lateral knee motion observed during a single-limb mini squat. BMC Musculoskelet Disord. 2010; 11(1): 265. https://doi.org/10.1186/1471-2474-11-265

14. Pantano KJ, White SC, Gilchrist LA, Leddy J. Differences in peak knee valgus angles between individuals with high and low $\mathrm{Q}$-angles during a single limb squat. Clin Biomech Bristol Avon. 2005; 20(9): 966-72. https://doi.org/10.1016/j.clinbiomech.2005.05.008

15. Ciesielska J, Lisiński P, Bandosz A, Huber J, Kulczyk A, Lipiec J. Hip strategy alterations in patients with history of low disc herniation and non-specific low back pain measured by surface electromyography and balance platform. Acta Bioeng Biomech Wroc Univ Technol. 2015; 17(3): 103-8

16. Zeller BL, McCrory JL, Kibler WB, Uhl TL. Differences in kinematics and electromyographic activity between men and women during the single-legged squat. Am J Sports Med. 2003; 31(3): 449-56. https://doi. org/10.1177/03635465030310032101

17. Flanagan S, Salem GJ, Wang M-Y, Sanker SE, Greendale GA. Squatting Exercises in Older Adults: Kinematic and Kinetic Comparisons. Med Sci Sports Exerc. 2003; 35(4): 635-43. https://doi.org/10.1249/01. MSS.0000058364.47973.06

18. Clément J, Hagemeister N, Aissaoui R, de Guise JA. Comparison of quasi-static and dynamic squats: a three-dimensional kinematic, kinetic and electromyographic study of the lower limbs. Gait Posture. 2014; 40(1): 94-100. https://doi.org/10.1016/j.gaitpost.2014.02.016

19. Hwang S, Kim Y, Kim Y. Lower extremity joint kinetics and lumbar curvature during squat and stoop lifting. BMC Musculoskelet Disord. 2009; 10: 15. https://doi.org/10.1186/1471-2474-10-15

20. Lamontagne M, Kennedy MJ, Beaulé PE. The Effect of Cam FAI on Hip and Pelvic Motion during Maximum Squat. Clin Orthop. 2009; 467(3): 645-50. https://doi.org/10.1007/s11999-008-0620-x

21. Macrum E, Bell DR, Boling M, Lewek M, Padua D. Effect of Limiting Ankle-Dorsiflexion Range of Motion on Lower Extremity Kinematics and Muscle-Activation Patterns during a Squat. J Sport Rehabil. 2012; 21(2): 144-50. https://doi.org/10.1123/jsr.21.2.144

22. Hwang S, Kim Y, Kim Y. Lower extremity joint kinetics and lumbar curvature during squat and stoop lifting. BMC Musculoskelet Disord. 2009; 10(1): 15. https://doi.org/10.1186/1471-2474-10-15

23. Zawadka M, Skublewska-Paszkowska M, Gawda P, Lukasik E, Smolka J, Jablonski M. What factors can affect lumbopelvic flexion-extension motion in the sagittal plane? A literature review. Hum Mov Sci. 2018; 58: 205-18. https://doi.org/10.1016/j.humov.2018.02.008

24. Pinto RZA, Souza TR, Trede RG, Kirkwood RN, Figueiredo EM, Fonseca ST. Bilateral and unilateral increases in calcaneal eversion affect pelvic alignment in standing position. Man Ther. 2008; 13(6): $513-9$.

25. Di Giulio I, Maganaris CN, Baltzopoulos V, Loram ID. The proprioceptive and agonist roles of gastrocnemius, soleus and tibialis anterior muscles in maintaining human upright posture. J Physiol. 2009; 587(Pt 10): 2399-416. https://doi.org/10.1113/jphysiol.2009.168690

26. Wikstrom EA, Tillman MD, Chmielewski TL, Cauraugh JH, Borsa PA. Dynamic postural stability deficits in subjects with self-reported ankle instability. Med Sci Sports Exerc. 2007; 39(3): 397-402. https:// doi.org/10.1249/mss.0b013e31802d3460

27. Hemmerich A, Brown H, Smith S, Marthandam SSK, Wyss UP. Hip, knee, and ankle kinematics of high range of motion activities of daily living. J Orthop Res Off Publ Orthop Res Soc. 2006; 24(4): 770-81. https://doi.org/10.1002/jor.20114

28. Powers CM. The influence of abnormal hip mechanics on knee injury: a biomechanical perspective. J Orthop Sports Phys Ther. 2010;40(2):4251. https://doi.org/10.2519/jospt.2010.3337

29. Reiman MP, Bolgla LA, Loudon JK. A literature review of studies evaluating gluteus maximus and gluteus medius activation during rehabilitation exercises. Physiother Theory Pract. 2012; 28(4): 257-68. https://doi.org/10.3109/09593985.2011.604981

30. Kasuyama T, Sakamoto M, Nakazawa R. Ankle Joint Dorsiflexion Measurement Using the Deep Squatting Posture. J Phys Ther Sci. 2009; 21(2): 195-9. https://doi.org/10.1589/jpts.21.195

31. Sung PS. A compensation of angular displacements of the hip joints and lumbosacral spine between subjects with and without idiopathic low back pain during squatting. J Electromyogr Kinesiol. 2013; 23(3): 741-5. https://doi.org/10.1016/j.jelekin.2013.02.003

32. Mitchell B, Bressel E, McNair PJ, Bressel ME. Effect of pelvic, hip, and knee position on ankle joint range of motion. Phys Ther Sport. 2008; 9(4): 202-8. https://doi.org/10.1016/j.ptsp.2008.08.002

33. Rabin A, Kozol Z, Spitzer E, Finestone AS. Weight-Bearing Ankle Dorsiflexion Range of Motion-Can Side-to-Side Symmetry Be Assumed? J Athl Train. 2015; 50(1): 30-5. https://doi.org/10.4085/1062$6050-49.3 .40$ 